\title{
Beautiful mirrors at the LHC
}

\author{
Kunal Kumar, ${ }^{a, b}$ William Shepherd, ${ }^{b, c}$ Tim M.P. Tait ${ }^{a, b, c}$ and Roberto Vega-Morales ${ }^{a, b}$ \\ ${ }^{a}$ HEP Division, Argonne National Lab, \\ Argonne IL 60439, U.S.A. \\ ${ }^{b}$ Northwestern University, \\ 2145 Sheridan Road, Evanston, IL 60208, U.S.A. \\ ${ }^{c}$ Department of Physics and Astronomy, University of California, \\ Irvine, CA 92697, U.S.A. \\ E-mail: kkumar@u.northwestern.edu, shepherd.william@uci.edu, \\ ttait@uci.edu, robertovegamorales2010@u.northwestern.edu
}

ABSTRACT: We explore the "Beautiful Mirrors" model, which aims to explain the measured value of $A_{F B}^{b}$, discrepant at the $2.9 \sigma$ level. This scenario introduces vector-like quarks which mix with the bottom, subtly affecting its coupling to the $Z$. The spectrum of the new particles consists of two bottom-like quarks and a charge $-4 / 3$ quark, all of which have electroweak interactions with the third generation. We explore the phenomenology and discovery reach for these new particles at the LHC, exploring single mirror quark production modes whose rates are proportional to the same mixing parameters which resolve the $A_{F B}^{b}$ anomaly. We find that for mirror quark masses $\lesssim 500 \mathrm{GeV}$, a $14 \mathrm{TeV}$ LHC with $300 \mathrm{fb}^{-1}$ is required to reasonably establish the scenario and extract the relevant mixing parameters.

KeYwords: Beyond Standard Model, Quark Masses and SM Parameters, Hadronic Colliders

ARXIV EPRINT: 1004.4895 


\section{Contents}

1 Introduction $\quad 1$

$2 \quad Z$ boson couplings to bottom quarks $\quad 3$

3 Beautiful mirrors $\quad 4$

3.1 Mixing and the mass eigenstates 4

3.2 Higgs couplings $\quad 6$

$3.3 W$ and $Z$ couplings $\quad 6$

$\begin{array}{lll}3.4 & \text { Sample parameters } & 7\end{array}$

4 Mirror quark phenomenology at the LHC $\quad 8$

4.1 Mirror quark production and decay 8

$\begin{array}{lll}4.2 & \bar{\chi} \text { production } & 10\end{array}$

$\begin{array}{ll}4.3 \text { Single } b_{2} / \overline{b_{2}} \text { production } & 11\end{array}$

4.4 Electroweak $b_{2}-\chi$ production 12

5 Conclusions $\quad 13$

\section{Introduction}

The primary mission of the Large Hadron Collider (LHC) is to seek evidence for the breakdown of the Standard Model (SM) [1]. For the most part, the SM with a light Higgs provides a very accurate description of the observed data coming from a wide variety of experiments. While deviations from the SM have come (and mostly gone), most disappear as statistics and experimental precision increases and theoretical inputs improve. The agreement between the SM predictions and experiment is unprecedented, particularly in the arena of precision electroweak measurements, many of which have per mil level uncertainties [2].

However, there is one notable exception. The forward-backward asymmetry of the bottom quark $\left(A_{F B}^{b}\right)$ shows roughly a $2.9 \sigma$ deviation $^{1}$ from the value predicted by a best fit to precision data within the SM [2]. While not in itself very significant, this deviation has persisted for more than a decade and may be a guide to what the LHC could find. $A_{F B}^{b}$ further plays an interesting role in the global fit to precision data, which in the context of the SM provides the indirect constraints on the Higgs mass [3, 4]. Indeed, the poor fit to $A_{F B}^{b}$ can be understood as a tension in the preferred value of $m_{h}$ between the leptonic observables, which prefer $m_{h} \sim 50 \mathrm{GeV}$ and $A_{F B}^{b}$ which prefers values closer to $\sim 1 \mathrm{TeV}$.

\footnotetext{
${ }^{1}$ It is interesting that recent Tevatron measurements also show an unexpected asymmetry in top quark pair production [5-7], though existing proposed new physics explanations do not typically correlate this with any particular effect on $A_{F B}^{b}[8-19]$.
} 
The fit has settled into an "unhappy" middle ground between the two, favoring the other measurements at the cost of disagreeing with the observed $A_{F B}^{b}$. As a result, if one simply ignores $A_{F B}^{b}$, one gets a fit to $m_{h}$ which is marginally at odds with the direct search bound from LEP-II [20].

Broadly defined, there are three attitudes one can take toward $A_{F B}^{b}$ and the precision data:

- One can assume $A_{F B}^{b}$ is a statistical (or unaccounted for systematic) effect and that reasonable variations of the other measured precision observables explain the tension between the SM fit to the Higgs mass and direct searches.

- One can consider the possibility that $A_{F B}^{b}$ itself does not reflect the presence of new physics, but accidently makes the SM fit to $m_{h}$ more palatable than it would otherwise have been. In this case, one can invoke new physics contributions to the Peskin and Takeuchi $T$ parameter [21] which may reconcile the indirect bounds on $m_{h}$ with the direct search limits (for a few examples, see [22-24]).

- One can take the attitude that the bottom quark couplings to the $Z$ boson may themselves reflect the presence of new physics [25-29].

In this article, we will take the last approach, and explore the consequences of one particular model of this kind, the "Beautiful Mirrors" model [27], which introduces new physics to produce the observed anomaly in $A_{F B}^{b}$.

The Beautiful Mirrors model works by introducing a new set of vector-like (or "mirror") quarks, which mix with the bottom quark, adjusting its coupling to the $Z$. Vector-like quarks are chosen so that gauge anomalies are trivially evaded, and the requirement that there be no source of EWSB other than the SM Higgs (motivated to avoid tree-level contributions to the oblique electroweak parameters) restricts the $S U(2)$ representations of the mirror quarks to singlets, triplets, and doublets. In [27], two versions of the doublet model were explored. The desired shift in the $Z$ couplings to bottom quarks may be effected for mirror quark quantum numbers under $(S U(3), S U(2), U(1))$ given by $(3,2,1 / 6)$ or $(3,2,-5 / 6)$. The first option looks like a vector-like fourth SM generation, and requires mirror quark masses $\lesssim 400 \mathrm{GeV}$ and SM Higgs mass $m_{h} \gtrsim 300 \mathrm{GeV}$ in order to fit the LEP data. Its detailed phenomenology was explored in ref. [28, 29]. The null results for direct searches for the mirror quarks [30] have severely restricted the parameter space of this model, leading us to consider the more exotic representation $(3,2,-5 / 6)$, which contains a bottom-like mirror quark $\omega$ and its electroweak partner, an electrically charged $-4 / 3$ quark, $\chi$. The precision data favors the masses for these "exotic mirrors" to be $\gtrsim 500 \mathrm{GeV}[27]$, making them perfect targets for a discovery at the LHC.

In this paper we explore the phenomenological consequences of the Exotic Mirrors model at the LHC. We begin in section 2 by revisiting the target $Z-b-\bar{b}$ couplings, which helps pin down the amount of mixing required when we discuss the beautiful mirrors model itself in section 3. LHC signals and strategies to establish a given signal as arising from the mirror quark solution to the $A_{F B}^{b}$ puzzle are presented in section 4 . We conclude in section 5 . 

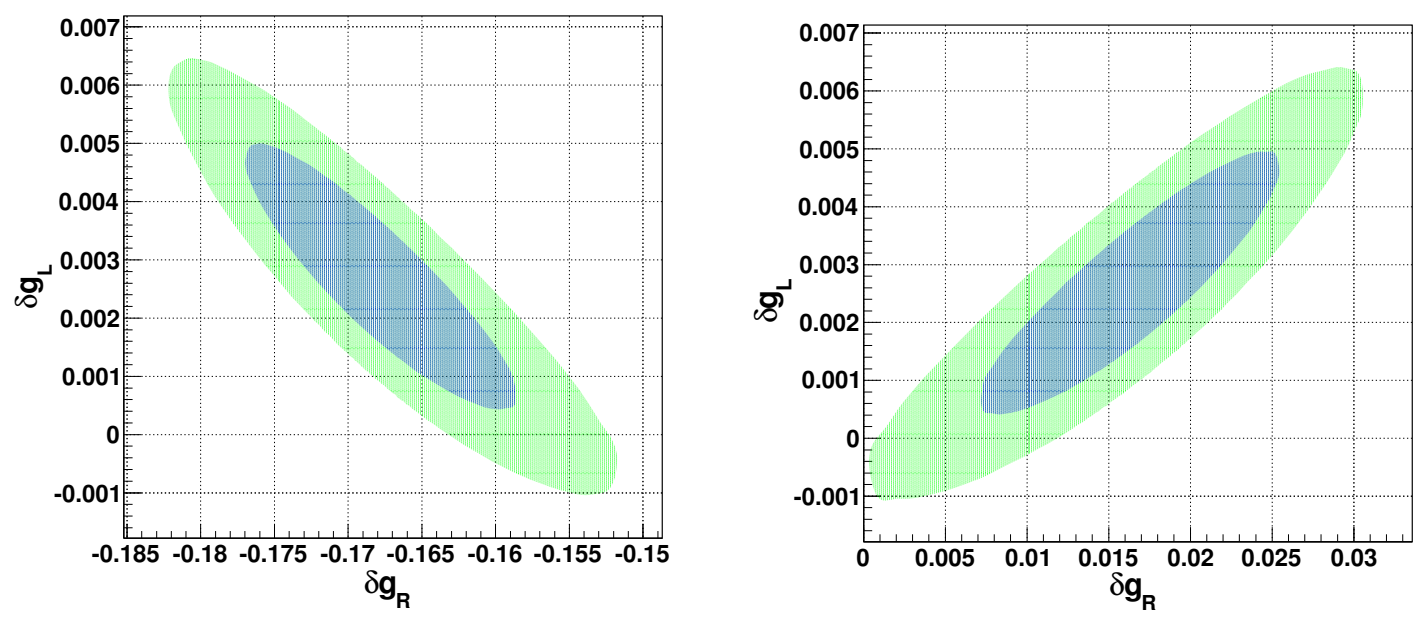

Figure 1. The regions in the $Z-b-\bar{b}$ coupling parameter space favored by EW precision data. The inner (outer) shaded regions correspond to $1 \sigma(2 \sigma)$ agreement with the best fit shifts in the leftand right-handed couplings.

\section{$2 \quad Z$ boson couplings to bottom quarks}

In this section, we examine the ranges of values of the $Z-b-\bar{b}$ couplings consistent with precision electroweak data. Modifications to bottom couplings must be applied subtly. While $A_{F B}^{b}$ is discrepant as described above, the branching ratio of $Z$ bosons decaying into $b \bar{b}$ (which is usually reported as a ratio between the decay to bottom quarks and into all hadrons, $R_{b} \equiv \Gamma(Z \rightarrow b \bar{b}) / \Gamma(Z \rightarrow$ hadrons $\left.)\right)$ shows no large deviation [2]. In addition, as discussed in [27], there is data from off of the $Z$-pole which, while less precise than the $Z$-pole measurements, implies important constraints on the signs of the couplings. In particular, the off-pole data requires that the left-handed interaction be close to the SM value, but does not restrict the sign of the right-handed value. Since together $A_{F B}^{b}$ and $R_{b}$ restrict the magnitude of the couplings, the allowed space of couplings lies within two disjoint regions of parameter space.

To explore the allowed regions of coupling space, we allow shifts in the left- and righthanded $Z-b-\bar{b}$ interactions by $\delta g_{L}$ and $\delta g_{R}$, respectively. We include these parameters in a global fit to the precision data, including the Tevatron measurements of the top and $W$ masses [31, 32]. We marginalize over $\alpha_{E M}, \alpha_{S}, m_{t}, m_{Z}$, and $m_{h}$, in particular allowing $m_{h}$ to take any value consistent with the direct search limit from LEP-II [20]. We assume there are no large additional contributions to the oblique parameters $S$ and $T$ beyond those which result from varying the top and Higgs masses. ${ }^{2}$ The results of the fit are presented in figure 1, which indicates that the data favors small $\left(\sim 10^{-3}\right)$ corrections to the left-handed coupling and more large (either $\sim+10^{-2}$ or $\sim-0.2$ ) shifts in $\delta g_{R}$. The values of $\delta g_{R}$ and $\delta g_{L}$ should be highly correlated with one another, in order to result in the necessary correction to $A_{F B}^{b}$, while the maximum and minimum changes are set by $R_{b}$.

\footnotetext{
${ }^{2}$ Note that the assumption of no large additional contributions to the $S$ and $T$ parameters is consistent with the exotic mirror scenario, but not with standard mirror quarks.
} 


\section{Beautiful mirrors}

The exotic beautiful mirrors model extends the Standard Model by introducing two sets of vector-like quarks, $\Psi_{L, R}$ with quantum numbers $(3,2,-5 / 6)$ and $\xi_{L, R}$ with quantum numbers $(3,1,-1 / 3)$. In terms of its $S U(2)$ components, $\Psi$ decomposes as,

$$
\Psi_{L, R}=\left(\begin{array}{c}
\omega_{L, R} \\
\chi_{L, R}
\end{array}\right)
$$

where $\omega$ is a charge $-1 / 3$ quark and $\chi$ has charge $-4 / 3$. Introducing vector-like quarks allows for new flavor mixing, which we will ultimately invoke to explain the measured value of $A_{F B}^{b}$. Among other effects, this mixing can lead to right handed $W$ couplings and tree level flavor changing interactions with the $Z$ and Higgs.

We assume for simplicity that the exotic quarks only couple to the third generation SM quarks, as $Z$ couplings to the two light generations appear to agree with SM predictions and any corrections are thus constrained to be small. Allowing for substantial mixing between the mirror quarks and the two lighter SM generations will generate tree level FCNC interactions which can contribute to $b \rightarrow s \gamma$ [44-47] which is highly constrained.

In addition, mixing with the light quarks leads to interactions of the type $Z$-b-s, $Z$ - $b-d$, and $Z-s-d$, as well as one loop box diagrams (with the mirror quark running in the loop), contributing to $B-\bar{B}$ [33-42] and possibly $K-\bar{K}$ and $D-\bar{D}$ mixing [43], all of which lead to tight constraints. These interactions are additionally constrained by rare decay processes of the strange and bottom mesons [48-51], as well as $B$ and $K$ meson decays such as $B \rightarrow \ell^{+} \ell^{-} X, B \rightarrow J / \psi K_{s}$ and $K \rightarrow \pi \nu \bar{\nu}[52-54]$.

That said, provided the mixing is small enough, the presence of such mixing between the mirror quarks and the first- and second-generation fermions (perhaps motivated by minimal flavor violation [55-59]) would not much affect the parameter space or resulting phenomenology. The choice of exotic mirrors (as opposed to the Standard Mirror gauge assignment) induces no right-handed $W$-t- $b$ interaction, evading potentially strong bounds again coming from $b \rightarrow s \gamma[60]$.

\subsection{Mixing and the mass eigenstates}

Electroweak symmetry breaking (EWSB) occurs as in the SM through the vacuum expectation value of a Higgs scalar, $\Phi$. We assume that the SM Higgs is the only source of EWSB, and write down the complete set of interactions between the mirror quarks and the third generation SM quarks, as allowed by $S U(3) \times S U(2) \times U(1)$ gauge invariance. In addition, the vector quarks have Dirac masses, whose magnitudes are not dictated by EWSB, but we will assume are at the $\sim \mathrm{TeV}$ scale. Such mass terms are protected by chiral symmetries, and thus technically natural in the sense of 't Hooft [61]. The complete set of Yukawa interactions and masses involving the mirror quarks are,

$$
\mathcal{L}_{\text {mass }}=-y_{1} \bar{Q}_{L}^{\prime} \Phi b_{R}^{\prime}-y_{R} \bar{\Psi}_{L}^{\prime} \tilde{\Phi} b_{R}^{\prime}-y_{L} \bar{Q}_{L}^{\prime} \Phi \xi_{R}^{\prime}-y_{5} \bar{\Psi}_{L}^{\prime} \tilde{\Phi} \xi_{R}^{\prime}-M_{2} \bar{\Psi}_{L}^{\prime} \Psi_{R}^{\prime}-M_{3} \bar{\xi}_{L}^{\prime} \xi_{R}^{\prime}+\text { h.c. }
$$

where the primed fields refer to gauge (as opposed to mass) eigenstates and $Q_{L}^{\prime}$ refers to the third generation quark doublet and $b_{R}^{\prime}$ is the third generation down-type singlet. 
After symmetry breaking, the couplings are most transparent in the unitary gauge, $\Phi=\frac{1}{\sqrt{2}}(0 v+h)^{T}$, where $v \sim 174 \mathrm{GeV}$ is the EWSB vacuum expectation value and $h$ is the Higgs boson. The mass and mixing terms of the Lagrangian may be written in matrix form,

$$
\mathcal{L}_{\text {mass }}=-\overline{\mathbf{d}}_{\mathbf{L}}^{\prime}\left(\mathbf{M}_{\mathbf{d}}+\frac{h}{v} \mathbf{N}_{\mathbf{d}}\right) \mathbf{d}_{\mathbf{R}}^{\prime}+\text { h.c. }
$$

where $\mathbf{d}_{\mathbf{L}, \mathbf{R}}^{\prime}=\left(b_{L, R}, \omega_{L, R}, \xi_{L, R}\right)$ are vectors in flavor space. $\mathbf{M}_{\mathbf{d}}$ is the bottom sector mass matrix,

$$
\mathbf{M}_{\mathbf{d}}=\left(\begin{array}{ccc}
Y_{1} & 0 & Y_{L} \\
Y_{R} & M_{2} & Y_{5} \\
0 & 0 & M_{3}
\end{array}\right)
$$

where $Y_{i}=y_{i} v / \sqrt{2} . \mathbf{N}_{\mathbf{d}} / v$ is the coupling matrix between the real Higgs and the down type quarks,

$$
\mathbf{N}_{\mathbf{d}}=\left(\begin{array}{ccc}
Y_{1} & 0 & Y_{L} \\
Y_{R} & 0 & Y_{5} \\
0 & 0 & 0
\end{array}\right) \text {. }
$$

To diagonalize the mass matrix we rotate by unitary matrices $\mathbf{U}_{\mathbf{d}}$ and $\mathbf{W}_{\mathbf{d}}$ which transform the left- and right-handed gauge eigenstates into the corresponding mass eigenstates (denoted by unprimed vectors in flavor space, $\mathbf{d}_{\mathbf{L}, \mathbf{R}}$ ). We parametrize these matrices

$$
\mathbf{U}_{\mathbf{d}}=\left(\begin{array}{ccc}
c_{12}^{L} c_{13}^{L} & s_{12}^{L} c_{13}^{L} & s_{13}^{L} \\
-s_{12}^{L} c_{23}^{L}-c_{12}^{L} s_{23}^{L} s_{13}^{L} & c_{12}^{L} c_{23}^{L}-s_{12}^{L} s_{23}^{L} s_{13}^{L} & s_{23}^{L} c_{13}^{L} \\
s_{12}^{L} s_{23}^{L}-c_{12}^{L} c_{23}^{L} s_{13}^{L} & -c_{12}^{L} s_{23}^{L}-s_{12}^{L} c_{23}^{L} s_{13}^{L} & c_{23}^{L} c_{13}^{L}
\end{array}\right)
$$

where $c_{12}^{L} \equiv \cos \theta_{12}^{L}$ and so on, and with an analogous expression for $\mathbf{W}_{\mathbf{d}}$ with $\theta_{i j}^{L} \rightarrow \theta_{i j}^{R}$. We have set potential phases to zero for simplicity; their inclusion will complicate the analysis slightly but are not expected to shed much light on the $A_{F B}^{b}$ puzzle. These matrices transform the gauge eigenstates to mass eigenstates,

$$
\begin{aligned}
\mathbf{d}_{\mathbf{L}}^{\prime} & =\mathbf{U}_{\mathbf{d}} \mathbf{d}_{\mathbf{L}}, \\
\mathbf{d}_{\mathbf{R}}^{\prime} & =\mathbf{W}_{\mathbf{d}} \mathbf{d}_{\mathbf{R}} .
\end{aligned}
$$


The requirement that these transformations produce the mass eigenbasis requires

$$
\mathbf{U}_{\mathbf{d}}^{\dagger} \mathbf{M}_{\mathbf{d}} \mathbf{W}_{\mathbf{d}}=\left(\begin{array}{ccc}
m_{1} & 0 & 0 \\
0 & m_{2} & 0 \\
0 & 0 & m_{3}
\end{array}\right) \text {. }
$$

For values of the mixing which are phenomenologically viable, $b_{1}$ is predominantly the original SM bottom quark fields, $b_{2}$ is mostly $\omega$ and $b_{3}$ is mostly $\xi$. The eigenvalues $m_{1} \equiv$ $m_{b}, m_{2}$, and $m_{3}$ are the bottom quark mass, and two exotic quark masses, respectively. Note that we do not necessarily order the exotic quarks $b_{2,3}$ by mass.

\subsection{Higgs couplings}

The Higgs couplings are complicated by the fact that the mass matrix receives contributions from the vector-like masses $M_{2}$ and $M_{3}$, resulting in flavor-violating Higgs couplings between the three mass eigenstate quarks,

$$
\mathcal{L}_{h q}=-\frac{h}{v} \overline{\mathbf{d}} \mathbf{V}_{\mathbf{d}} P_{R} \mathbf{d}+h . c .
$$

where $\mathbf{V}_{\mathbf{d}}=\mathbf{U}_{\mathbf{d}}^{\dagger} \mathbf{N}_{\mathbf{d}} \mathbf{W}_{\mathbf{d}}$. The off diagonal entries of $\mathbf{V}_{\mathbf{d}}$ will lead to tree level flavor changing couplings between the Higgs of the form $h-\bar{b}_{1}-b_{2}$, etc. Such couplings allow for decays of the heavy quarks into a bottom quark and a Higgs, as discussed below.

\section{3 $W$ and $Z$ couplings}

We now examine the modifications to the $W$ and $Z$ couplings coming from the mixing of the bottom quark with the exotics. In the mass basis there are $W$ couplings of the form

$$
\mathcal{L}_{W}=\frac{g}{\sqrt{2}} W_{\mu}^{-}\left[\bar{\chi} \gamma^{\mu}\left(\mathbf{U}_{\mathbf{d}}^{2 \mathbf{j}} P_{L}+\mathbf{W}_{\mathbf{d}}^{2 \mathbf{j}} P_{R}\right) \mathbf{d}^{\mathbf{j}}+\overline{\mathbf{d}}^{\mathbf{i}} \gamma^{\mu} \mathbf{U}_{\mathbf{d}}^{1 \mathbf{i} *} P_{L} t\right]+h . c .
$$

where $g=e / \cos \theta_{w}$ as usual.

The couplings between the $Z$ and the down-type quarks may be written in matrix form,

$$
\mathcal{L}_{Z}=\frac{g}{\cos \theta_{w}} Z_{\mu} \overline{\mathbf{d}} \gamma^{\mu}\left(\mathbf{L} P_{L}+\mathbf{R} P_{R}\right) \mathbf{d}+\text { h.c. }
$$

where

$$
\begin{aligned}
\mathbf{L} & =\mathbf{U}_{\mathbf{d}}^{\dagger} \mathbf{g}_{\mathbf{L}} \mathbf{U}_{\mathbf{d}}, \\
\mathbf{R} & =\mathbf{W}_{\mathbf{d}}^{\dagger} \mathbf{g}_{\mathbf{R}} \mathbf{W}_{\mathbf{d}},
\end{aligned}
$$

and the $\mathbf{g}_{\mathbf{L}, \mathbf{R}}$ are diagonal matrices in the gauge basis with left and right-handed couplings of the down-type quarks to the $Z$ boson as their entries,

$$
\begin{aligned}
& \mathbf{g}_{\mathbf{L}}=\operatorname{Diag}\left(-\frac{1}{2}+\frac{1}{3} \sin ^{2} \theta_{w}, \quad \frac{1}{2}+\frac{1}{3} \sin ^{2} \theta_{w}, \frac{1}{3} \sin ^{2} \theta_{w}\right), \\
& \mathbf{g}_{\mathbf{R}}=\operatorname{Diag}\left(\frac{1}{3} \sin ^{2} \theta_{w}, \quad \frac{1}{2}+\frac{1}{3} \sin ^{2} \theta_{w}, \quad \frac{1}{3} \sin ^{2} \theta_{w}\right) .
\end{aligned}
$$


Our primary concern is to modify the $b$-quark couplings wth the $Z$, in order to explain the measured $A_{F B}^{b}$ while remaining consistent with $R_{b}$. These couplings are determined by the 11 entries of the $\mathbf{L}$ and $\mathbf{R}$ matrices. In terms of the parameterization, eq. (3.6), these entries are,

$$
\begin{aligned}
\mathbf{L}^{11} & =\mathbf{g}_{\mathbf{L}}{ }^{11}\left(c_{12}^{L} c_{13}^{L}\right)^{2}+\mathbf{g}_{\mathbf{L}}{ }^{22}\left(-s_{12}^{L} c_{23}^{L}-s_{13}^{L} s_{23}^{L} c_{12}^{L}\right)^{2}+\mathbf{g}_{\mathbf{L}}{ }^{33}\left(s_{12}^{L} s_{23}^{L}-s_{13}^{L} c_{23}^{L} c_{12}^{L}\right)^{2}, \\
\mathbf{R}^{11} & =\mathbf{g}_{\mathbf{R}}{ }^{11}\left(c_{12}^{R} c_{13}^{R}\right)^{2}+\mathbf{g}_{\mathbf{R}}{ }^{22}\left(-s_{12}^{R} c_{23}^{R}-s_{13}^{R} s_{23}^{R} c_{12}^{R}\right)^{2}+\mathbf{g}_{\mathbf{R}}{ }^{33}\left(s_{12}^{R} s_{23}^{R}-s_{13}^{R} c_{23}^{R} c_{12}^{R}\right)^{2}(3.1
\end{aligned}
$$

These expressions may be simplified by noting that the term proportional to the electric charge is common to all of the diagonal entries of $\mathbf{g}_{\mathbf{L}, \mathbf{R}}$ and thus cancels out of shifts in the coupling, leaving behind only the non-universal terms proportional to $T_{3}$. In terms of the mixing angles, these shifts become,

$$
\begin{aligned}
\delta g_{L}^{b} & =\frac{g}{2 \cos \theta_{w}}\left[1-\left(c_{12}^{L} c_{13}^{L}\right)^{2}+\left(s_{12}^{L} c_{23}^{L}+s_{13}^{L} s_{23}^{L} c_{12}^{L}\right)^{2}\right], \\
\delta g_{R}^{b} & =\frac{g}{2 \cos \theta_{w}}\left(s_{12}^{R} c_{23}^{R}+s_{13}^{R} s_{23}^{R} c_{12}^{R}\right)^{2} .
\end{aligned}
$$

\subsection{Sample parameters}

Comparing the expressions for the shifts in the $Z-b-\bar{b}$ interactions in Equation (3.17) with the results in figure 1 , we can determine relations among the input parameters which will improve the agreement of $A_{F B}^{b}$ with its measured value.

We will analyze a specific point within this parameter space and examine the collider phenomenology. For simplicity we assume negligible mixing between $\omega$ and $\xi$ with $s_{12}^{R}=$ 0.21 and $s_{13}^{L}=0.078$ and all other mixing angles set to zero. For greater clarity of notation, therefore, we denote these angles simply by $s_{R}$ and $s_{L}$ henceforth. The negligible mixing between $\omega$ and $\xi$ means we have chosen $Y_{5}=0$. The Yukawa couplings in the bottom sector mass matrix can be related to the mixing angles in the limit of negligible $m_{b}$ as follows

$$
y_{L} \simeq \frac{M_{3} s_{L}}{v} \quad y_{R} \simeq \frac{M_{2} s_{R}}{v}
$$

The mixings which are postulated here yield couplings shifts through equation (3.17) of

$$
\delta g_{L}^{b}=2.27 \times 10^{-3} \quad \delta g_{R}^{b}=1.64 \times 10^{-2}
$$

and were chosen with the intent of simplifying our discussion by removing the mixings not relevant to the measured asymmetry while giving coupling shifts near the center of the favored region.

These assumptions leave us with only three free parameters in our system, $m_{b_{2}}, m_{b_{3}}$, and $m_{h}$. The relation between $m_{b_{2}}$ and $m_{\chi}$ is

$$
m_{\chi}^{2}=m_{b_{2}}^{2}-Y_{R}^{2}=m_{b_{2}} c_{R}^{2}
$$

As mentioned in [27] the fit to data is not very sensitive to $m_{b_{3}}$ as long as it is below a few TeV. Looking at the $m_{h}-m_{\chi}$ parameter space plot, in the study just mentioned, we choose $m_{h}=120 \mathrm{GeV}$ and let the masses $m_{b_{2}}$ (or equivalently $m_{\chi}$ ) and $m_{b_{3}}$ vary between $500 \mathrm{GeV}$ and $2 \mathrm{TeV}$. These points lie within $1 \sigma$ of the best fit point. We study the detailed phenomenology at this point in parameter space, but will note where interesting deviations are possible. 


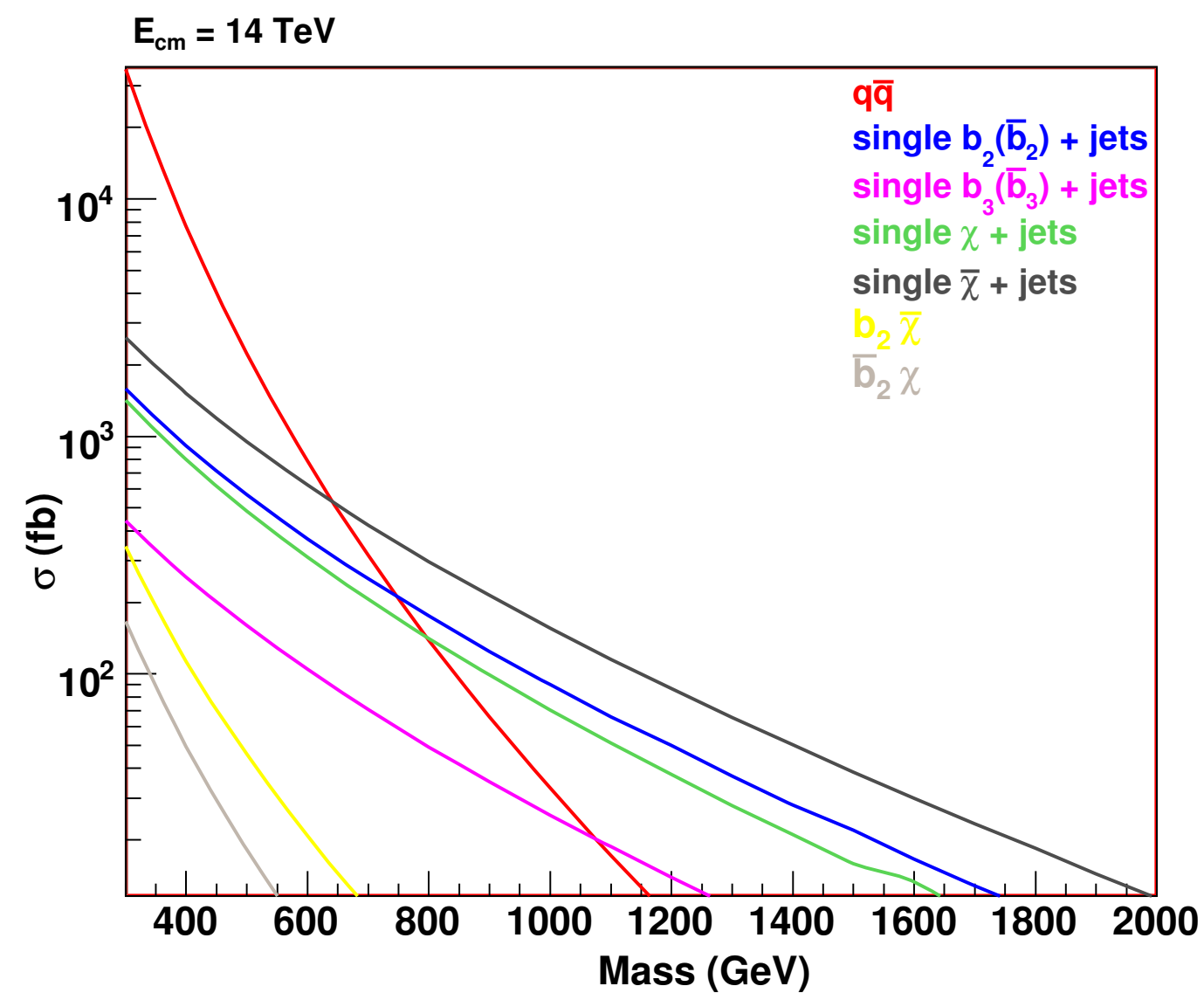

Figure 2. Production cross sections for both single and pair production of mirror quarks, as a function of their masses and for the mixing angles specified in the text. The single $b_{2,3}+$ jets rates sum both production of $b_{2,3}+$ jets and $\bar{b}_{2,3}+$ jets. For this plot we have chosen $m_{h}=120 \mathrm{GeV}$

\section{Mirror quark phenomenology at the LHC}

The key question for the LHC is whether or not the mirror quarks can be discovered, and their $S U(2) \times U(1)$ gauge representations and mixing angles understood well enough to experimentally connect them to the measured value of $A_{F B}^{b}$. This task is complicated by the fact that the mixing through EWSB itself obscures the original representations of $b_{2}$ and $b_{3}$, and the $\chi$, while unusual in that it has charge $-4 / 3$, decays into $W^{-} b$, looking much like a $t^{\prime}$ which produces a "wrong sign" bottom quark in its decay; measuring the charge of the final state $b$ quark is extremely subtle, though perhaps not impossible [62,63].

\subsection{Mirror quark production and decay}

The mirror quarks $\chi, b_{2}$, and $b_{3}$ can be produced either in pairs through QCD, or singly, through the electroweak interaction. Single $\chi$ quarks are produced through a $b q$ initial state with a $t$-channel $W$ boson exchanged whereas single $b_{2}$ and $b_{3}$ arise from a $b q$ initial state with a $t$-channel $Z$ boson (or, to a much smaller degree, Higgs boson) exchanged. The 
resulting cross sections as a function of the mass of the exotic quark in question are plotted in figure 2, where we have used the mixing angles appropriate for the sample solution to the $A_{F B}^{b}$ puzzle discussed in section 3.4 and a Higgs mass of $120 \mathrm{GeV}$ (although the results are quite robust for larger Higgs masses as well). The cross sections have been computed at tree level with the MadEvent code [64], using the CTEQ6L parton distribution functions (PDFs) [65]. As can be seen, for the modest mixing angles favored by $A_{F B}^{b}$, pair production is the dominant mechanism for exotic quark masses below $\sim 700 \mathrm{GeV}$. The difference in rates between single $\chi\left(Q=-\frac{4}{3}\right)$ and single $\bar{\chi}\left(Q=+\frac{4}{3}\right)$ production can be understood from the difference in PDFs of the initial state quarks. The $\bar{\chi}$, which is primarily produced from an initial state $u$-quark, is expected to have a higher electroweak production rate than $\chi$ which which comes primarily from an initial $d$-quark. The same trend is familiar from single top production in the Standard Model.

Pair production cross sections are not affected by the choice of mixing angles at all, and thus the prediction of that cross section is robust for any point in the parameter space of the exotic mirrors model. Single production cross sections are proportional to the square of the relevant mixing angle or combination of mixing angles, and thus those cross sections will be shifted by changes in mixing angles.

The $\chi$ quark decays with $100 \%$ branching ratio into $W^{-} b$ for our parameter point, appearing as a a $\bar{t}^{\prime}$ which produces $b$ instead of a $\bar{b}$ when it decays. As such, it is sensitive to the usual fourth generation $t^{\prime}$ searches at the LHC, with an expected reach through pair production of roughly $800 \mathrm{GeV}[66]$ for $100 \mathrm{fb}^{-1}$ of integrated luminosity at $14 \mathrm{TeV}$. Searches for $t^{\prime}$ quarks at the Tevatron provide bounds of $m_{\chi} \geq 335 \mathrm{GeV}$ [67]. Allowing $\omega-\xi$ mixing can open additional channels for $\chi$ decay such as $\chi \rightarrow W^{-} b_{3}$, subject to kinematic constraints. Depending on the size of the mixing this can become comparable in magnitude to the direct decay to purely SM final states.

The bottom-like quarks $b_{2}$ and $b_{3}$ can decay into $Z b_{i}, h b_{i}$ (provided the Higgs is light enough), $W t$, and $W \chi$. For our example parameter point, the $\chi$ is too heavy to be produced on-shell in decays of $b_{2}$, and $b_{3}$ does not have a charged-current coupling to $\chi$ due to our choice of no $\omega-\xi$ mixing. Heavy quark decays into $W t$ are rendered negligible by this assumption as well. Thus, these quarks decay only through the FCNC modes,

$$
\begin{aligned}
& b_{2,3} \rightarrow h b, \\
& b_{2,3} \rightarrow Z b .
\end{aligned}
$$

For Higgs mass of $120 \mathrm{GeV}$ and exotic quark mass of $500 \mathrm{GeV}$, the branching ratios for both $b_{2}$ and $b_{3}$ are $52 \% b_{2,3} \rightarrow Z b$ and $48 \% b_{2,3} \rightarrow h b$. Note that these branching ratios are insensitive to changes in the mixing angles $s_{L, R}$, and are not strongly sensitive to increases in exotic quark mass. The $Z$ decay mode offers the possibility of lepton pairs in the final state (with modest branching ratio) whereas the $h$ decay mode leads to a $b_{2,3} \rightarrow b \bar{b} b$ final state a large fraction of the time.

Branching ratios of $b_{i}$ decays are independent of shifts in $s_{L}$ and $s_{R}$ in absence of $\omega-\xi$ mixing. This is because in that limit the flavor changing couplings of the higgs and the $Z$ both have identical dependence on the mixing angles. If mixing between the new vectorlike quarks is allowed it can lead to shifts in the relative branching fractions to $Z$ and $h$ 
final states, and also opens additional decay channels, such as $b_{3} \rightarrow W+\chi$ or $b_{2} \rightarrow Z b_{3}$, dependent on kinematic constraints. These will in general lead to more spectacular cascade decays, as the decay product vector quark then further decays to SM fields.

While the LHC cannot hope to exclude the entire range of mirror quark masses favored by the electroweak fit, it is sensitive to much of the parameter space. For masses $\lesssim 1 \mathrm{TeV}$, we can expect based on earlier studies [66] that by the end-running of the LHC (which we take to be at center-of-mass running of $14 \mathrm{TeV}$ and data sets on the order of $300 \mathrm{fb}^{-1}$ ), the LHC will have observed the mirror quarks through pair production in the decay modes $\chi \rightarrow$ $W^{-} b$ and $b_{2,3} \rightarrow Z b \rightarrow \ell^{+} \ell^{-} b$. We explore several subdominant production processes which can help differentiate the beautiful mirrors model from other models with additional vectorlike quarks, and establish the mixing parameters as consistent with a solution to $A_{F B}^{b}$. We choose as a reference value for our studies mirror quark masses $M_{2}\left(=m_{\chi}\right)=500 \mathrm{GeV}$. Such masses are consistent with Tevatron bounds and within the $1 \sigma$ fit to the precision data [27], and represent a cautiously optimistic region of parameter space. We assume $M_{3}$, which is not well constrained by the fit, is $\geq 1 \mathrm{TeV}$, and thus do not assume $b_{3}$ will be observable.

\section{$4.2 \bar{\chi}$ production}

The process $u \bar{b} \rightarrow d \bar{\chi}$ is the largest of the single production modes in the model, and under our assumption that $M_{3} \gg M_{2}$, its rate is proportional to $s_{R}^{2}$, thus providing a measure of the key mixing which is responsible for $\delta g_{R}$. There is also a contribution from the lefthanded mixing, but this is constrained to be small by precision data. We attempt to extract the signal (and thus measure $s_{R}$ ) by looking at the semi-leptonic $\chi$ decay: $p p \rightarrow j \bar{\chi} \rightarrow j b \ell \nu$ where $j$ is a light-quark initiated jet and $\ell=e$ or $\mu$. To improve background rejection, we do not attept to reconstruct single $\chi$ production here, focusing on the dominant single $\bar{\chi}$ signal. For our sample parameter point, the signal inclusive cross section, including branching ratios, is $949 \mathrm{fb}$. The SM background (with very mild acceptance cuts on the jets) is $12.7 \mathrm{nb}$, dominantly $W j j$ production, with smaller contributions from $t \bar{t}$ and single top production. Events are showered and hadronized with PYTHIA [68], and we estimate detector effects with PGS [69] using the default LHC detector model of MadEvent.

To separate the signal from the background efficiently, we require that the event contain exactly one $b$-tagged jet with transverse momentum $P_{T} \geq 100 \mathrm{GeV}$, one positively-charged lepton with $P_{T} \geq 50 \mathrm{GeV}$ (which is sufficient to trigger on the events even in high luminosity running), no more than two jets with $P_{T} \geq 30 \mathrm{GeV}$, and missing momentum $\mathbb{E}_{T} \geq 50 \mathrm{GeV}$. We further require that the invariant mass of the two highest $P_{T}$ jets $M_{j j}$ be $\geq 100 \mathrm{GeV}$. We assume the $\not_{T}$ arises from a neutrino present in a on-shell $W$ decay, and use the $W$ mass to reconstruct the longitudinal neutrino momentum. Armed with that information, we can reconstruct the four-momentum for the $W$ boson, which we combine with the $b$-tagged jet to form the invariant mass which in a signal event would reconstruct the $\chi$ mass of $500 \mathrm{GeV}$. We apply a wide cut to this quantity, requiring it to be in the range $400-600 \mathrm{GeV}$.

We found a signal acceptance of about $1 \%$ and a background suppression factor of $2.6 \times 10^{-5}$ using these cuts. While the signal-to-background ratio remains small, sufficient statistics can be generated for a significant observation of the process and a measurement of the signal cross section. With $100 \mathrm{fb}^{-1}$ of data the total number of expected events 
exceeds the SM prediction by 950 , equivalent to $5.2 \sigma$, constituting a discovery of the single production process for $\bar{\chi}$, and with $300 \mathrm{fb}^{-1}$ the cross section can be measured to be $949 \pm 147 \mathrm{fb}$, where systematic uncertainties in determining the acceptances are assumed to be small in comparison to the large statistical uncertainties. This measurement corresponds to 2,800 expected signal events over 99,000 expected background events. Extracting $s_{R}$ from the cross section is straightforward, as all other quantities entering the conversion are known with effectively zero error compared to the measured cross section. We end up with a measurement of $s_{R}^{2}=0.044 \pm 0.007$.

The discovery potential in this channel extends well beyond the mass studied here. Azuelos et al [71] found that a vector like $t^{\prime}$ was discoverable through the $t^{\prime} \rightarrow W b$ channel up to $m_{t^{\prime}} \simeq 2.5 \mathrm{TeV}$. Our model predicts an identical signal, with comparable production cross section and more favorable branching ratio for this measurement.

The fact that $\chi$ has charge $-4 / 3$ is a very distinctive feature compared to other models of vector-like quarks, but difficult to establish experimentally. One could attempt to measure the charge of the $b$ quark produced in a $\chi$ decay; this has been successfully employed by Tevatron experiments to establish the top quark charge [62,63], but depends sensitively on modeling the detector response correctly, and thus is beyond the scope of this work. Additional strategies could be to examine processes such as $\chi \bar{\chi} \gamma$, which is expected to lead to a successful LHC measurement of the top quark charge [70]. We have performed simulations of $\chi \bar{\chi} \gamma$ production, but find that the contribution induced by photon radiation from the parent quark becomes lost in radiation from the $W$ or lepton in its decay. The large $\chi$ mass has the unfortunate effect of both reducing the over-all rate substantially compared to the $t \bar{t} \gamma$, and also collimates the $\chi$ decay products, making it more difficult to extract the cases where the photon is radiated by the final state lepton from that where it is radiated from the quark itself than was true for the well-spread out top quark decay products.

Ultimately, the most promising argument for the charge of the $\chi$ may be indirect by the failure to observe the decay mode $\chi \rightarrow Z t$, which would generically be present for a charge $2 / 3$ vector quark, which would be allowed to mix with the top. This argument rests on the assumption that one has observed $b_{2} \rightarrow Z b$, and thus knows that the newly discovered objects are in fact vector-like as opposed to chiral quarks. However, it is worth bearing in mind that even for a vector-like $t^{\prime}$, the $Z-t^{\prime}-t$ interaction is controlled by separate mixing angles from those in the $b$ sector, and thus may turn out to be very small. ${ }^{3}$

\subsection{Single $b_{2} / \overline{b_{2}}$ production}

After single $\bar{\chi}$ production, the next largest single mirror quark production mode is single $b_{2}$ production (including single $\bar{b}_{2}$ production), which proceeds through an FCNC $Z$ or $h$ exchange in the $t$-channel. The rate for this process is proportional to $s_{R}^{2} c_{R}^{2}$, and thus provides another measurement of the mixing angle $s_{R}^{2}$. We examine the feasibility of observing the process $p p \rightarrow j b_{2} \rightarrow j b \ell^{+} \ell^{-}$through an intermediate $Z$ boson from the $b_{2}$ decay (and also the conjugate process for $\bar{b}_{2}$ ). The signal cross section (including branching ratios) for $m_{b_{2}}=500 \mathrm{GeV}$ is $16.6 \mathrm{fb}$. The background is dominantly $Z b \bar{b}$ and $t \bar{t}$ and is 125 pb after acceptance cuts.

\footnotetext{
${ }^{3}$ In fact, the reasonable agreement between the experimental measurements of $b \rightarrow s \gamma$ and SM predictions requires that the product of the $t-t^{\prime}$ and $b-b^{\prime}$ mixings be $\lesssim 10^{-2}[60]$.
} 
We require at least one of the leptons in the event to have $P_{T} \geq 20 \mathrm{GeV}$, sufficient for triggering. We select events with at least one $b$-tagged jet with $P_{T} \geq 70 \mathrm{GeV}$. The main criteria to distinguish between the signal and background are the reconstructed mass of two leptons, which should be close to the $Z$ mass and its combination with the $b$-jet to form an invariant mass close to $m_{2}$. In events with more than one $b$-tagged jet (as is often the case for the background processes), we combine the $b$-jet that has the largest $P_{T}$ with the lepton pair to form the reconstructed $b_{2}$ mass. We require the reconstructed $b_{2}$ mass to be within a $25 \mathrm{GeV}$ window of the reference value of $m_{2}=500 \mathrm{GeV}$. This window contains $42.3 \%$ of the signal rate after jet smearing. In addition, we place a restriction on the angular separation of $\Delta R \leq 1$ between the pair of leptons, since the signal produces highly boosted $Z$ bosons from the $b_{2}$ decay whose decay products are collimated.

At an integrated luminosity of $300 \mathrm{fb}^{-1}$ the number of predicted signal events for our parameter point is 280 , after applying the above mentioned cuts. The analysis efficiency is $5.6 \%$. The number of expected background events is 1260 after background suppression by a factor of $3.4 \times 10^{-5}$. The resulting significance is $7.9 \sigma$, and the measurement of the mixing angle is $s_{R}^{2}=0.044 \pm 0.008$, which is comparable to the precision offered by single $\bar{\chi}$ production. While not a direct reconstruction of the vector-like quarks, these two measurements together provide evidence that the primary mixing with the third generation SM quarks is through the bottom sector, with no apparent mixing involving the top (which is forbidden in our construction by $U(1)_{E M}$ but could be allowed in generic models of mirror quarks containing top-like objects).

\subsection{Electroweak $b_{2}-\chi$ production}

Another process which allows us to extract information about the mixing angle is electroweak production of a pair of mirror quarks, $\chi b_{2}$ through an $s$-channel $W$ boson. The cross section, which is proportional to $c_{R}^{2}$, turns out to be quite small due to the fact that on top of being governed by weak couplings, two heavy quarks are being produced. To analyze this signal we look at the process $p p \rightarrow \chi b_{2} \rightarrow \ell^{ \pm} \ell^{\mp} b \bar{b} \ell^{\prime} \nu$ (and its charge-conjugate version). This particular signature has the $b_{2}$ decaying through a $Z$ into $\ell^{+} \ell^{-}$whereas $\chi$ decays as usual into $W b$. After acceptance cuts, the cross section for this signal is $0.359 \mathrm{fb}$. The background was generated using MadEvent and the relevant decays were obtained with BRIDGE [72] before showering and hadronizing with PYTHIA. Again detector effects were estimated with PGS using the default LHC detector model of MadEvent. This resulted in a background cross section of $3.8 \mathrm{fb}$.

Due to the small number of events, distinguishing signal from background is difficult. In order to retain enough events to obtain sufficient statistics one must be conservative in applying cuts. We first require that the event contain two $b$-tagged jets with $P_{T} \geq 60$ $\mathrm{GeV}$. Since we expect the charged lepton pair decaying from the highly boosted $\mathrm{Z}$ to be collimated we first find the $\ell^{ \pm} \ell^{\mp}$ pair with the smallest $\Delta R$ and combine it with one of the $b$ jets to form an invariant mass (in a signal event this would reconstruct the $b_{2}$ mass) which we require to be greater than $50 \mathrm{GeV}$.

To determine which lepton is associated with the neutrino we again assume the $\mathbb{E}_{T}$ arises from a neutrino present in an on-shell $W$ decay. We then find any charged leptons not 
belonging to the pair which decay from the $\mathrm{Z}$ and out of those find the one with the smallest $\Delta \phi$ relative to the missing $E_{T}$. We use the $W$ mass to reconstruct the longitudinal neutrino momentum, and from that we reconstruct the four-momentum for the $W$ boson and combine it with the other $b$-tagged jet with $P_{T} \geq 60 \mathrm{GeV}$ to obtain the invariant mass (in a signal event this would reconstruct the $\chi$ mass) which we require to be greater than $200 \mathrm{GeV}$.

With a signal acceptance of $6.25 \%$ these selection criteria lead to an expected 10 signal events at $300 \mathrm{fb}^{-1}$. We expect 3 background events after a suppression factor of $2.4 \times 10^{-3}$ leading to a significance of $4 \sigma$ for the signal over background. While this does not constitute a discovery, when combined with the information obtained from other signals, it provides evidence that the $\chi$ and $b_{2}$ quarks form an $S U(2)$ doublet, and thus does help to verify the structure of the Beautiful mirrors model.

\section{Conclusions}

While the possibilities for discovery at the LHC are vast, it may be that there are clues as to what could be discovered in the form of modest deviations already present in lower energy data. In this article, we have discussed one such deviation, the forward-backward asymmetry of the bottom quark, which has persisted for more than a decade and appears to play a key role in the SM fit to the Higgs mass. We have explored one particular model which addresses the discrepancy by adding additional vector-like quarks which mix with the $b$, subtly affecting its coupling to the $Z$ boson.

These quarks are perfect targets for discovery at the LHC, which is likely to initially observe them through pair production. We have examined the prospects for observing single production as well. While single production has smaller rates, being suppressed by electroweak strength couplings and mixing angles, it probes the basic phenomena responsible for the solution to the mystery of $A_{F B}^{b}$. In particular, we have studied single $\bar{\chi}$ production followed by the dominant decay $\bar{\chi} \rightarrow W^{+} \bar{b}$, single $b_{2}$ production followed by the decay $b_{2} \rightarrow Z b \rightarrow \ell^{+} \ell^{-} b$, and pair production of $\chi \bar{b}_{2}$ (with the same decay chains as above). These processes are expected to be visible for quark masses up to about $500 \mathrm{GeV}$ at a $14 \mathrm{TeV} \mathrm{LHC}$ with hundreds of $\mathrm{fb}^{-1}$, and provide evidence that the $\chi$ and $b_{2}$ quarks form a vector-like electroweak doublet which mixes primarily with the bottom quark. The primary mixing parameter, $s_{R}^{2}$, responsible for explaining the value of $A_{F B}^{b}$, can be measured at the $20 \%$ level.

\section{Acknowledgments}

The authors are grateful for conversations with Carlos E.M. Wagner. T Tait appreciates the SLAC theory group for their extraordinary generosity during his many visits. KK and R V-M would like to thank Gabe Shaughnessy, Jamie Gainer, and Patrick Fox for helpful discussions. Research at Argonne National Laboratory is supported in part by the Department of Energy under contract DE-AC02-06CH11357.

Open Access. This article is distributed under the terms of the Creative Commons Attribution Noncommercial License which permits any noncommercial use, distribution, and reproduction in any medium, provided the original author(s) and source are credited. 


\section{References}

[1] D.E. Morrissey, T. Plehn and T.M.P. Tait, New Physics at the LHC, arXiv:0912.3259 [SPIRES].

[2] LEP Electroweak Working Group, Precision Electroweak Measurements and Constraints on the Standard Model, LEPEWWG/2009-01.

[3] M.S. Chanowitz, Electroweak Data and the Higgs Boson Mass: A Case for New Physics, Phys. Rev. D 66 (2002) 073002 [hep-ph/0207123] [SPIRES].

[4] M.S. Chanowitz, The $Z \rightarrow$ anti- $b$ b decay asymmetry: Lose-lose for the standard model, Phys. Rev. Lett. 87 (2001) 231802 [hep-ph/0104024] [SPIRES].

[5] D0 collaboration, V.M. Abazov et al., First measurement of the forward-backward charge asymmetry in top quark pair production, Phys. Rev. Lett. 100 (2008) 142002 [arXiv:0712.0851] [SPIRES].

[6] CDF collaboration, T. Aaltonen et al., Forward-Backward Asymmetry in Top Quark Production in $p \bar{p}$ Collisions at sqrts $=1.96$ TeV, Phys. Rev. Lett. 101 (2008) 202001 [arXiv:0806.2472] [SPIRES].

[7] CDF collaboration, Measurement of the Forward-Backward Asymmetry in Top Pair Production in 3.2/fb of ppbar collisions at $\sqrt{s}=1.96 \mathrm{TeV}$, Conf. Note 9724 (March 2009) http://www-cdf.fnal.gov/physics/new/top/2009/tprop/Afb/.

[8] O. Antunano, J.H. Kuhn and G. Rodrigo, Top quarks, axigluons and charge asymmetries at hadron colliders, Phys. Rev. D 77 (2008) 014003 [arXiv:0709.1652] [SPIRES].

[9] S. Jung, H. Murayama, A. Pierce and J.D. Wells, Top quark forward-backward asymmetry from new t-channel physics, Phys. Rev. D 81 (2010) 015004 [arXiv:0907.4112] [SPIRES].

[10] J.M. Arnold, M. Pospelov, M. Trott and M.B. Wise, Scalar Representations and Minimal Flavor Violation, JHEP 09 (2010) 073 [arXiv: 0911.2225] [SPIRES].

[11] P.H. Frampton, J. Shu and K. Wang, Axigluon as Possible Explanation for $p \bar{p} \rightarrow t \bar{t}$ Forward-Backward Asymmetry, Phys. Lett. B 683 (2010) 294 [arXiv:0911.2955] [SPIRES].

[12] J. Shu, T.M.P. Tait and K. Wang, Explorations of the Top Quark Forward-Backward Asymmetry at the Tevatron, Phys. Rev. D 81 (2010) 034012 [arXiv:0911.3237] [SPIRES].

[13] A. Arhrib, R. Benbrik and C.-H. Chen, Forward-backward asymmetry of top quark in diquark models, arXiv:0911.4875 [SPIRES].

[14] P. Ferrario and G. Rodrigo, Heavy colored resonances in top-antitop + jet at the LHC, JHEP 02 (2010) 051 [arXiv:0912.0687] [SPIRES].

[15] I. Dorsner, S. Fajfer, J.F. Kamenik and N. Kosnik, Light colored scalars from grand unification and the forward-backward asymmetry in top quark pair production, Phys. Rev. D 81 (2010) 055009 [arXiv:0912.0972] [SPIRES].

[16] D.-W. Jung, P. Ko, J.S. Lee and S.-h. Nam, Model independent analysis of the forward-backward asymmetry of top quark production at the Tevatron, Phys. Lett. B 691 (2010) 238 [arXiv:0912.1105] [SPIRES].

[17] J. Cao, Z. Heng, L. Wu and J.M. Yang, Top quark forward-backward asymmetry at the Tevatron: a comparative study in different new physics models, Phys. Rev. D 81 (2010) 014016 [arXiv:0912.1447] [SPIRES]. 
[18] V. Barger, W.-Y. Keung and C.-T. Yu, Asymmetric Left-Right Model and the Top Pair Forward- Backward Asymmetry, Phys. Rev. D 81 (2010) 113009 [arXiv:1002.1048] [SPIRES].

[19] Q.-H. Cao, D. McKeen, J.L. Rosner, G. Shaughnessy and C.E.M. Wagner, Forward-Backward Asymmetry of Top Quark Pair Production, Phys. Rev. D 81 (2010) 114004 [arXiv:1003.3461] [SPIRES].

[20] A. Ludwig, Results of beyond the standard model Higgs searches from the LEP experiments, hep-ex/0610088 [SPIRES].

[21] M.E. Peskin and T. Takeuchi, Estimation of oblique electroweak corrections, Phys. Rev. D 46 (1992) 381 [SPIRES].

[22] M.E. Peskin and J.D. Wells, How can a heavy Higgs boson be consistent with the precision electroweak measurements?, Phys. Rev. D 64 (2001) 093003 [hep-ph/0101342] [SPIRES].

[23] G. Altarelli, F. Caravaglios, G.F. Giudice, P. Gambino and G. Ridolfi, Indication for light sneutrinos and gauginos from precision electroweak data, JHEP 06 (2001) 018 [hep-ph/0106029] [SPIRES].

[24] M.S. Carena, E. Ponton, T.M.P. Tait and C.E.M. Wagner, Opaque branes in warped backgrounds, Phys. Rev. D 67 (2003) 096006 [hep-ph/0212307] [SPIRES].

[25] H.E. Haber and H.E. Logan, Radiative corrections to the $Z$ b anti-b vertex and constraints on extended Higgs sectors, Phys. Rev. D 62 (2000) 015011 [hep-ph/9909335] [SPIRES].

[26] A. Djouadi, G. Moreau and R.K. Singh, Kaluza-Klein excitations of gauge bosons at the LHC, Nucl. Phys. B 797 (2008) 1 [arXiv:0706.4191] [SPIRES].

[27] D. Choudhury, T.M.P. Tait and C.E.M. Wagner, Beautiful mirrors and precision electroweak data, Phys. Rev. D 65 (2002) 053002 [hep-ph/0109097] [SPIRES].

[28] D.E. Morrissey and C.E.M. Wagner, Beautiful mirrors, unification of couplings and collider phenomenology, Phys. Rev. D 69 (2004) 053001 [hep-ph/0308001] [SPIRES].

[29] J.L. Chkareuli, I.G. Gogoladze and A.B. Kobakhidze, Natural nonSUSY SU(N) GUTs, Phys. Lett. B 340 (1994) 63 [SPIRES].

[30] CDF collaboration, Search for HEavy Top $t \prime \rightarrow W q$ in Lepton Plus Jets Events in $\int L d t=4.6 \mathrm{fb}^{-1}$, http://www-cdf.fnal.gov/physics/new/top/2010/tprop/Tprime_v46_public/ public_4.6/tprime_CDFnotePub.pdf .

[31] Tevatron Electroweak Working Group collaboration, Combination of CDF and DO Results on the Mass of the Top Quark, arXiv:0903.2503 [SPIRES].

[32] Tevatron Electroweak Working Group collaboration, Updated Combination of CDF and DO Results for the Mass of the W Boson, arXiv:0908.1374 [SPIRES].

[33] J. Maalampi and M. Roos, Flavor mixing in the presence of a fourth down quark, Phys. Lett. B 188 (1987) 487 [SPIRES].

[34] Y. Nir and D.J. Silverman, Z mediated flavor changing neutral currents and their implications for CP asymmetries in $B^{0}$ decays, Phys. Rev. D 42 (1990) 1477 [SPIRES].

[35] D. Silverman, $Z$ mediated $B-\bar{B}$ mixing and $B$ meson $C P$-violating asymmetries in the light of new FCNC bounds, Phys. Rev. D 45 (1992) 1800 [SPIRES]. 
[36] L. Lavoura and J.P. Silva, Bounds on the mixing of the down type quarks with vector - like singlet quarks, Phys. Rev. D 47 (1993) 1117 [SPIRES].

[37] W.-S. Choong and D. Silverman, New phases in CP-violating B decay asymmetries from mixing to singlet down quarks, Phys. Rev. D 49 (1994) 2322 [SPIRES].

[38] V.D. Barger, M.S. Berger and R.J.N. Phillips, Quark singlets: Implications and constraints, Phys. Rev. D 52 (1995) 1663 [hep-ph/9503204] [SPIRES].

[39] D. Silverman, B factory constraints on isosinglet down quark mixing and predictions for other CP-violating experiments, Int. J. Mod. Phys. A 11 (1996) 2253 [hep-ph/9504387] [SPIRES].

[40] G. Barenboim, F.J. Botella and O. Vives, Tree level FCNC in the B system: From CP asymmetries to rare decays, Phys. Rev. D 64 (2001) 015007 [hep-ph/0012197] [SPIRES].

[41] D. Hawkins and D. Silverman, Iso-singlet down quark mixing and CP-violation experiments, Phys. Rev. D 66 (2002) 016008 [hep-ph/0205011] [SPIRES].

[42] J.A. Aguilar-Saavedra, Effects of mixing with quark singlets, Phys. Rev. D 67 (2003) 035003 [hep-ph/0210112] [SPIRES].

[43] T. Yanir, Phenomenological constraints on extended quark sectors, JHEP 06 (2002) 044 [hep-ph/0205073] [SPIRES].

[44] G. Bhattacharyya, G.C. Branco and D. Choudhury, Radiative B decay as a test of CKM unitarity, Phys. Lett. B 336 (1994) 487 [Erratum ibid. B 340 (1994) 266] [hep-ph/9406424] [SPIRES].

[45] L.T. Handoko and T. Morozumi, $b \rightarrow s(d)$ gamma with a vector - like quark as fourth generation, Mod. Phys. Lett. A 10 (1995) 309 [Erratum ibid. A 10 (1995) 1733] [hep-ph/9409240] [SPIRES].

[46] C.-H.V. Chang, D. Chang and W.-Y. Keung, Vector quark model and B meson radiative decay, hep-ph/9811354 [SPIRES].

[47] A.L. Kagan and M. Neubert, QCD anatomy of $B \rightarrow X_{s} \gamma$ decays, Eur. Phys. J. C 7 (1999) 5 [hep-ph/9805303] [SPIRES].

[48] G.C. Branco, T. Morozumi, P.A. Parada and M.N. Rebelo, CP asymmetries in B0 decays in the presence of flavor changing neutral currents, Phys. Rev. D 48 (1993) 1167 [SPIRES].

[49] M.R. Ahmady, M. Nagashima and A. Sugamoto, Inclusive dileptonic rare B decays with an extra generation of vector-like quarks, Phys. Rev. D 64 (2001) 054011 [hep-ph/0105049] [SPIRES].

[50] M.R. Ahmady, M. Nagashima and A. Sugamoto, $B \rightarrow X_{s} \ell^{+} \ell^{-}$in the vectorlike quark model, hep-ph/0108007 [SPIRES].

[51] T. Morozumi, Z.H. Xiong and T. Yoshikawa, $B \rightarrow X_{s} \ell^{+} \ell^{-}$and $B \rightarrow K \pi$ decays in vectorlike quark model, hep-ph/0408297 [SPIRES].

[52] G. Barenboim, F.J. Botella and O. Vives, Constraining models with vector - like fermions from FCNC in K and B physics, Nucl. Phys. B 613 (2001) 285 [hep-ph/0105306] [SPIRES].

[53] H.-Y. Cai and D.-X. Zhang, $b \rightarrow s s \bar{d}$ in a vector quark model, Commun. Theor. Phys. 44 (2005) 887 [hep-ph/0410144] [SPIRES].

[54] M.R. Ahmady, F. Falahati and S.M. Zebarjad, Charmless hadronic two-body $B_{s}$ decays within the vector quark model, Acta Phys. Polon. B 40 (2009) 2775 [SPIRES]. 
[55] G. D'Ambrosio, G.F. Giudice, G. Isidori and A. Strumia, Minimal flavour violation: An effective field theory approach, Nucl. Phys. B 645 (2002) 155 [hep-ph/0207036] [SPIRES].

[56] V. Cirigliano, B. Grinstein, G. Isidori and M.B. Wise, Minimal flavor violation in the lepton sector, Nucl. Phys. B 728 (2005) 121 [hep-ph/0507001] [SPIRES].

[57] A.J. Buras, P. Gambino, M. Gorbahn, S. Jager and L. Silvestrini, Universal unitarity triangle and physics beyond the standard model, Phys. Lett. B 500 (2001) 161 [hep-ph/0007085] [SPIRES].

[58] R.S. Chivukula and H. Georgi, Composite Technicolor Standard Model, Phys. Lett. B 188 (1987) 99 [SPIRES].

[59] L.J. Hall and L. Randall, Weak scale effective supersymmetry, Phys. Rev. Lett. 65 (1990) 2939 [SPIRES].

[60] F. Larios, M.A. Perez and C.P. Yuan, Analysis of $t b W$ and $t t Z$ couplings from CLEO and LEP/SLC data, Phys. Lett. B 457 (1999) 334 [hep-ph/9903394] [SPIRES].

[61] G. 't Hooft, C. Itzykson, A. Jaffe, H. Lehmann, P.K. Mitter, I.M. Singer and R. Stora, Recent Developments In Gauge Theories, in Proceedings, Nato Advanced Study Institute, Cargese, France, August 26 - September 8 (1979), New York U.S.A. Plenum (1980) 438 p, Nato Advanced Study Institutes Series: Series B, Physics, 59.

[62] D0 collaboration, V.M. Abazov et al., Experimental discrimination between charge 2 e/3 top quark and charge 4 e/3 exotic quark production scenarios, Phys. Rev. Lett. 98 (2007) 041801 [hep-ex/0608044] [SPIRES].

[63] CDF collaboration, Z. Gunay Unalan, First CDF measurement of the top quark charge using the top decay products, Nucl. Phys. Proc. Suppl. 177-178 (2008) 297 [SPIRES].

[64] J. Alwall et al., MadGraph/MadEvent v4: The New Web Generation, JHEP 09 (2007) 028 [arXiv:0706.2334] [SPIRES].

[65] J. Pumplin et al., New generation of parton distributions with uncertainties from global QCD analysis, JHEP 07 (2002) 012 [hep-ph/0201195] [SPIRES].

[66] J.A. Aguilar-Saavedra, Identifying top partners at LHC, JHEP 11 (2009) 030 [arXiv: 0907.3155] [SPIRES].

[67] CDF collaboration, A. Lister, Search for Heavy Top-like Quarks $t \prime \rightarrow W q$ Using Lepton Plus Jets Events in 1.96 TeV p pbar Collisions, arXiv:0810.3349 [SPIRES].

[68] T. Sjöstrand, S. Mrenna and P.Z. Skands, PYTHIA 6.4 Physics and Manual, JHEP 05 (2006) 026 [hep-ph/0603175] [SPIRES].

[69] http://www.physics.ucdavis.edu/ conway/research/software/pgs/pgs4-general.htm.

[70] U. Baur, M. Buice and L.H. Orr, Direct measurement of the top quark charge at hadron colliders, Phys. Rev. D 64 (2001) 094019 [hep-ph/0106341] [SPIRES].

[71] G. Azuelos et al., Exploring little Higgs models with ATLAS at the LHC, Eur. Phys. J. C 39S2 (2005) 13 [hep-ph/0402037] [SPIRES].

[72] P. Meade and M. Reece, BRIDGE: Branching ratio inquiry/decay generated events, hep-ph/0703031 [SPIRES]. 